\title{
JAK/STAT3 regulated global gene expression dynamics during late-stage reprogramming process
}

\author{
Ling Wang ${ }^{1 \dagger}$, Zongliang Jiang ${ }^{1,4+}$, Delun Huang ${ }^{1,2}$, Jingyue Duan ${ }^{1}$, Chang Huang ${ }^{1}$, Shannon Sullivan ${ }^{1}$, Kaneha Vali ${ }^{1}$, \\ Yexuan Yin ${ }^{1}$, Ming Zhang ${ }^{2}$, Jill Wegrzyn ${ }^{3}$, Xiuchun ( Cindy) Tian ${ }^{1 *}$ and Young Tang ${ }^{1 *}$ (D)
}

\begin{abstract}
Background: The generation of induced pluripotent stem cells (iPSCs) has underdefined mechanisms. In addition, leukemia inhibitory factor (LIF) activated Janus kinase/signal transducer and activator of transcription 3 (JAK/STAT3) pathway is the master regulator for naïve-state pluripotency achievement and maintenance. However, the regulatory process to attain naïve pluripotent iPSCs is not well understood.

Results: We performed transcriptome analysis to dissect the genomic expression during mouse iPSC induction, with or without blocking the JAK/STAT3 activity. We describe JAK/STAT3 signaling-specific biological events such as gametogenesis, meiotic/mitotic cell cycle, and DNA repair, and JAK/STAT3-dependent expression of key transcription factors such as the naïve pluripotency-specific genes, developmental pluripotency associated (Dppa) family, along with histone modifiers and non-coding RNAs in reprogramming. We discover that JAK/STAT3 activity does not affect early phase mesenchymal to epithelial transition (MET) but is necessary for proper imprinting of the DIk1-Dio3 region, an essential event for pluripotency achievement at late-reprogramming stage. This correlates with the JAK/STAT3dependent stimulation of Dppa3 and Polycomb repressive complex 2 (PRC2) genes. We further demonstrate that JAK STAT3 activity is essential for DNA demethylation of pluripotent loci including Oct4, Nanog, and the D/k1-Dio3 regions. These findings correlate well with the previously identified STAT3 direct targets. We further propose a model of pluripotency achievement regulated by JAK/STAT3 signaling during the reprogramming process.
\end{abstract}

Conclusions: Our study illustrates novel insights for JAK/STAT3 promoted pluripotency establishment, which are valuable for further improving the naïve-pluripotent IPSC generation across different species including humans.

Keywords: Reprogramming, IPSC, LIF, STAT3, Pluripotency, Gametogenesis, DIk1-Dio3, Imprinting, DNA methylation

\section{Background}

Generation of induced pluripotent stem cells (iPSCs) represents a powerful way to establish embryonic stem cell (ESC)-like cells through ectopic expression of the four transcription factors, namely Oct4, Klf4, Sox2, and $c-M y c$ (OKSM) [1]. However, its mechanism is not completely understood. This hinders further effort to improve the reprogramming efficiency and general safety of human iPSCs for clinical applications. Early mechanistic studies revealed that a mesenchymal to epithelial

\footnotetext{
*Correspondence: xiuchun.tian@uconn.edu; yong.tang@uconn.edu ${ }^{\dagger}$ Equal contributors

'Department of Animal Science, Institute for Systems Genomics, University of Connecticut, Storrs, CT, USA

Full list of author information is available at the end of the article
}

transition (MET) is required for successful reprogramming [2, 3]. Large-scale transcriptome and epigenomic analysis further revealed a multi-step reprogramming process, where somatic cells undergo an initiation/MET phase, followed by an intermediate phase characterized by stochastic activation of pluripotent markers and transient upregulation of developmental genes. Subsequently, the reprogrammed cells enter a late maturation/ stabilization phase hallmarked by silencing of transgenes and activation of core pluripotent circuitry, to form completely reprogrammed, pluripotent iPSCs [3-7]. The entire reprogramming process is also characterized by epigenetic changes such as histone $\mathrm{H} 3$ lysine $(\mathrm{K})$ acetylation and methylation, DNA demethylation or de novo 
methylation, to activate the core pluripotency genes, and poise reprogrammed cells for differentiation under developmental cues $[4,6,8,9]$. However, to date, a complete understanding to pluripotency establishment at latereprogramming stage has not been achieved.

The transition of somatic to pluripotent state is also regulated by stage-specific expression of non-coding RNAs such as microRNAs (miRNAs) $[4,8,10,11]$ and long intervening non-coding RNAs (lincRNAs) [9, 1214], to regulate the expression of pro-differentiation and metabolic processes. The activation of maternally expressed lincRNA cluster Gtl2-Rian-Mirg, localized in the Dlk1-Dio3 region at chromosome 12qF1 (Additional file 1), is essential for full pluripotency in mouse iPSC generation. Improper imprinting of this region is associated with poor chimera capacity of iPSCs and compromised generation of viable iPSC-mice by tetraploid complementation [15-17]. The expression of the Gtl2-Rian-Mirg is controlled by the intergenic differential methylated region (IG-DMR) localized between $D l k 1$ and Gtl2 genes [18] (Additional file 1). This region is hypermethylated at late-reprogramming stage [15], and only a small portion of iPSCs could re-establish proper imprinting of this region ( 50\% methylated IG-DMR) and become truly pluripotent $[16,17]$. Vitamin $C$ or the developmental pluripotency associated 3 (Dppa3) gene antagonize the binding of de novo DNA methyltransferases 3 (Dnmt3s) to IG-DMR region, therefore prevent the IG-DMR hypermethylation in reprogramming [15, 19]. Polycomb repressive complex 2 (PRC2) also antagonize Dnmt3s for proper imprinting of Dlk1-Dio3 in mouse ESCs [20]. However, how Dppa3 or PRC2 activity is controlled in reprogramming to ensure proper imprinting of the Dlk1-Dio3 region is unclear.

The cytokine leukemia inhibitory factor (LIF) activates Janus kinas/signal transducer and activator of transcription 3 (JAK/STAT3) pathway by inducing heterodimerization of LIF receptor and the signal transducer protein gp130 [21, 22]. Activation of JAK/STAT3 by LIF ensures naïve-state mouse ESC pluripotency and self-renewal [23-27]. STAT3 also plays a key role in naïve-state iPSC generation [28-30]. However, the question remains how exactly JAK/STAT3 activity regulates different biological events to ensure complete reprogramming. Characterization of JAK/STAT3 mediated reprogramming activities is needed to fully elucidate its downstream mechanism/effectors for naïve-state pluripotency generation. Such knowledge will also help to improve the LIF signal-dependent naïve-state iPSC generation across different species including humans [31].

We previously showed that enhancing STAT3 activity in reprogramming promotes pluripotency establishment from mouse embryonic fibroblasts (MEFs), while blocking JAK/STAT3 activity only leads to partially reprogrammed pre-iPSCs [29]. These pre-iPSCs failed to silence the OKSM transgenes and to activate key pluripotent genes such as Oct4 and Nanog [29], two hallmarks of late-stage reprogramming [3-7]. To further understand the regulatory role of JAK/STAT3 in latestage reprogramming, we performed transcriptome analysis to those reprogrammed cells at two different time points, and identified biological events specific to JAK/ STAT3 signaling. We further discovered that JAK/ STAT3 regulates proper activation of the imprinted Dlk1-Dio3 region in reprogramming. Our study unveils novel mechanisms for LIF/STAT3 regulated late-stage reprogramming process.

\section{Results}

RNA-seq analysis reveals dynamic global gene expression between two different reprogramming stages regulated by JAK/STAT3 activity

We performed transcriptome analysis of the RNA samples of reprogrammed MEFs that carry a GFP reporter controlled by the Oct4 distal enhancer region (OGMEFs), as described previously [29] (Fig. 1, GEO accession number GSE97261). Briefly, OG-MEFs were seeded on day minus one (D-1), transduced with retroviral OKSM on the next day (D0) and then cultured in LIFcontaining reprogramming medium, with the addition of either control DMSO $(\mathrm{Ctl})$ or $1 \mu \mathrm{M}$ specific Jak inhibitor I (Jaki) [32, 33] starting on Day 3 (D3). RNAs were extracted from the D18 reprogrammed cells (named DMSO-Stage 1 (S1) or Jaki-S1, respectively), or from induced colonies picked on D21 and expanded one more passage (p2) (named DMSO-Stage 2 (S2) or Jaki-S2, respectively) (Fig. 1). We chose these two time points (S1 and S2) to identify global gene expression differences between $\mathrm{Ctl}$ and Jaki-treatments, since the GFP positive $(\mathrm{GFP}+)$ colonies (indication of endogenous Oct4 activation) in Ctl reprogrammed cells started to develop quickly between $\mathrm{S} 1$ and S2, while those colonies in Jakitreatment remained GFP negative (GFP-) [29]. Pearson correlation coefficient and clustering analysis of all detected transcripts by RNA-seq (FPKM >0.1) illustrated significant difference in global gene expression patterns between the S1 and S2 reprogrammed cells (Fig. 1b, c). These data show that dynamic change of global gene expression happened between S1 and S2. In addition, clustering analysis classified the Jaki- and DMSO-treated cells into different groups within each stage (Fig. 1c). We also performed principle component analysis (PCA) to all detected genes across our samples. Plots using the two most significant principle components further confirmed the differences between $\mathrm{S} 1$ and $\mathrm{S} 2$ reprogrammed cells, and between the DMSO Ctl and Jaki-treated cells within each stage (Fig. 1d). Thus, S1 and S2 samples represent reprogrammed cells at two 
a

C
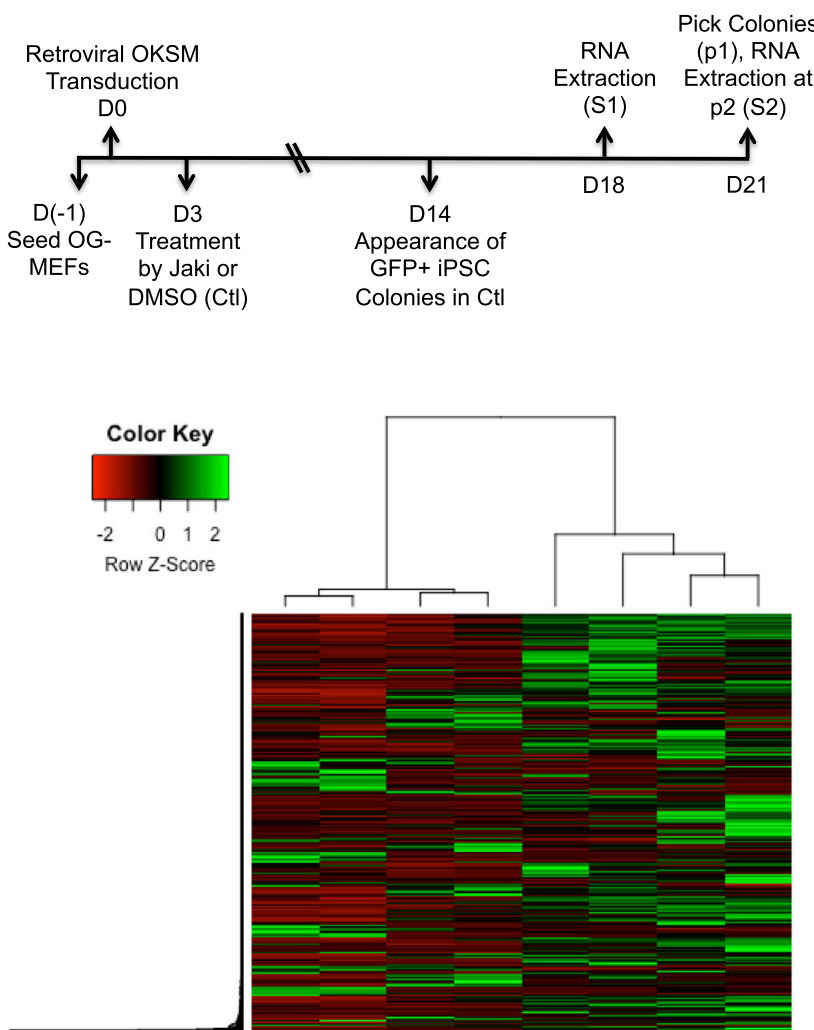

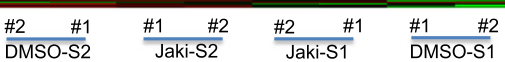

b

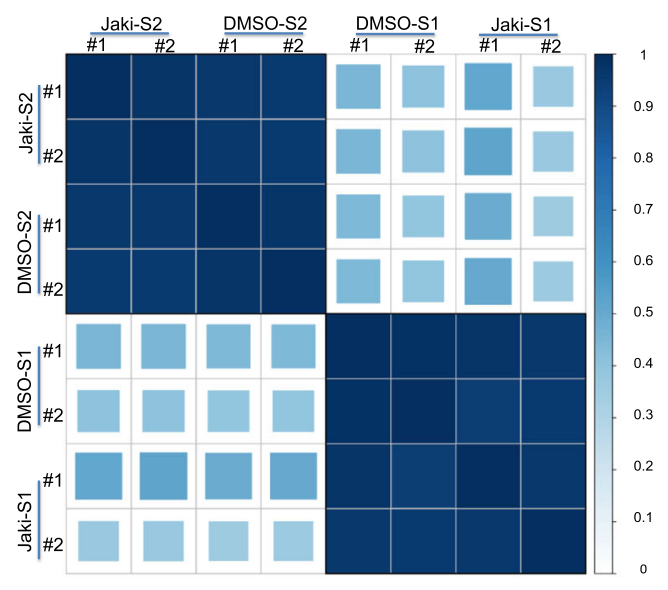

d

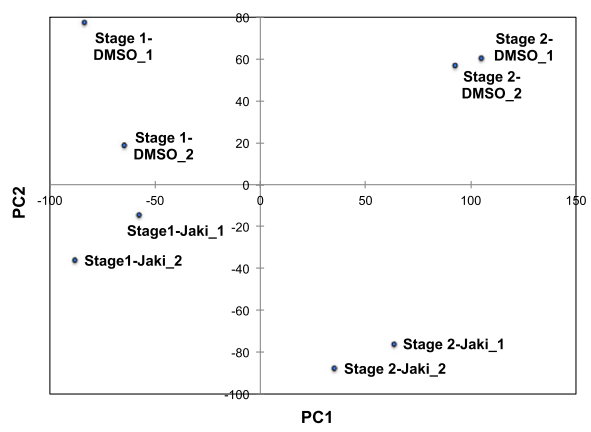

e

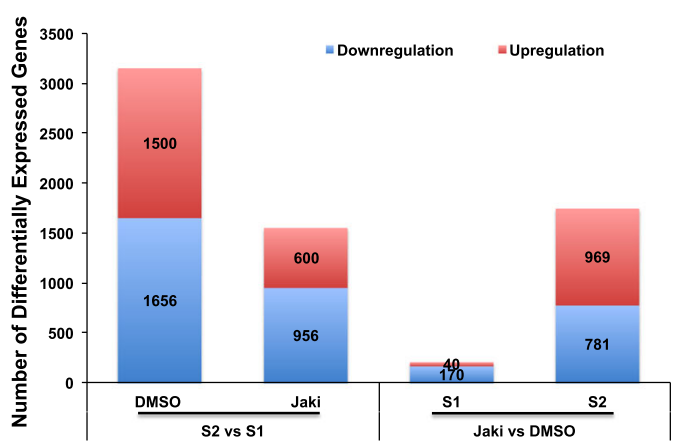

Fig. 1 Dynamic Gene Expression Changes at Two Different Reprogramming Stages. a Schematic diagram depicting the reprogramming process and dates for RNA sample collection from reprogrammed cells. $\mathbf{b}$ Pearson Correlation of the duplicated samples of different reprogramming conditions and stages. The colored bar along the right side of the heatmap indicates the Pearson's correlation coefficient. c Hierarchical clustering of differentially expressed genes among different treatments and reprogramming stages. The relative abundance is represented by color (red, lower abundance; green, higher abundance), as indicated by the color key. d PCA analysis to the transcriptomes of different reprogramming samples. PC1 and PC2 represent the top two dimensions of the differentially expressed genes. e Bar chart representing the numbers of up- or down-regulated DEGs between S1 and S2 and between two treatments at the same reprogramming stage 
distinct stages, and that inhibiting JAK/STAT3 activity significantly impacts global gene expression patterns at either stage.

We further analyzed the differentially expressed genes (DEGs) either 1) between the S1 and S2 reprogrammed cells within each treatment, to compare the dynamic reprogramming differences in Ctl (undisturbed JAK/STAT3 signaling) and Jaki (blocked JAK/STAT3 signaling) conditions, or 2) between the Jaki- and DMSO-treatments at S1 or S2, to identify specific targets of JAK/STAT3 activity at these two reprogramming stages. Out of the 13,547 genes detected, Cuffdiff analysis revealed the largest numbers of significantly up-/down-regulated genes (1500/1656, fold change $>1.62 \times$ ) happened in Ctl reprogramming between S2 and S1 (Fig. 1e, Additional file 2). Whereas the smallest numbers of significantly up-/down-regulated genes (40/ 170) were found between the Jaki- and DMSO-treatment at S1, there are 969/781 up-/down-regulated DEGs identified at S2 (Fig. 1e, Additional file 2). The sharp contrast in the numbers of DEGs at S1 and S2 between Jaki vs. DMSO-treatment supports the notion that JAK/STAT3 plays a more significant role for pluripotency establishment at late-reprogramming stage, and correlates with the previous reports that STAT3 functions for naïve-state induction from pre-iPSCs and primed-state epiblast stem cells, as well as for the self-renewal of ESCs [29, 34-36].

\section{JAK/STAT3 regulates specific biological events between the two reprogramming stages}

We then asked how JAK/STAT3 signaling specifically regulates the reprogramming events. For all significantly upregulated DEGs from S1 to S2 in either Ctl- or Jakitreatment, 351 were commonly upregulated under both treatments, 1149 were specifically upregulated in Ctl reprogramming, while 249 genes were upregulated only in Jaki-treatment (Fig. 2a-left). These common or specific DEGs were subject to gene ontology (GO) analysis using the DAVID platform [37], with similar GO-terms for biological processes (BPs, false discovery rate $(\mathrm{FDR})<0.05)$ grouped together to illustrate reprogramming events under these conditions (Additional file 3). Multiple upregulated events from S1 to S2 common for both Jaki- and Ctl-treatments were identified (Fig. 2b-left). These include the protein translation, redox process, nucleosome assembly/transcription regulation, and negative regulation of megakaryocyte differentiation. The latter two BPs are characterized by upregulation of genes from various histone subfamilies, including $H 1 H 1, H 2 B 1, H 2 B 2$, $H 3 A 1, H 41$, and H44 (Additional file 3). On the other hand, activation of events like mitotic cell cycle, spermatogenesis/meiotic cell cycle, and the DNA repair process that is intrinsically associated with different phases of cell cycle [38], are only observed in Ctl reprogramming (Fig. 2b-right, Additional file 3). The protein modification processes were also upregulated in Ctl reprogramming, and over-represented by DEGs either for protein folding, such as the $F K B P$ family (FKBP3-6, - 11) and the CCT family $(C C T 2-4,-6 A$, -7) [39, 40], or for protein sumoylation, such as Sumo1, Sumo2, and the E3 SUMO-protein ligase Pias2 [41] (Fig. 2b-right, Additional file 3). However, no significant GO-term was found from the 249 upregulated DEGs under Jaki-treatment (Fig. 2a-left, and data not shown).

For all significantly downregulated DEGs between S2 and S1 reprogrammed cells, 481 genes were commonly downregulated in both Ctl- and Jaki-treatments, while 1175 and 475 genes were specifically downregulated in Ctl- or Jaki-treatment, respectively (Fig. 2a-right, Additional file 3). GO-analysis of those groups of DEGs revealed commonly downregulated biological events from $\mathrm{S} 1$ to S2 reprogramming in both $\mathrm{Ctl}-$ and Jakitreatments (Fig. 2c-top). These include cell adhesion and migration, positive regulation of transcription, cell differentiation such as endo-/meso-dermal development, VEGF signaling, response to estradiol, and cell proliferation. However, the downregulation of cellular immune response and protein phosphorylation process from S1 to S2 can only be observed in Ctl- but not Jakitreatment (Fig. 2c-lower left and right, Additional file 3). Thus, these data reveal that multiple biological events associated with undisturbed JAK/STAT3 signaling happen during reprogramming. These include the upregulation of gametogenesis, meiotic/mitotic cell cycle, and protein modification including protein folding and sumoylation, and downregulation of immune responses and protein phosphorylation.

\section{JAK/STAT3 is critical for activation of gametogenesis and meiotic cell cycle event genes in reprogramming}

To further evaluate the biological events in reprogramming that are JAK/STAT3-specific, we compared the DEGs between Jaki- and Ctl-treatments at the same reprogramming stage. As there are limited numbers of significant DEGs identified at S1 (Fig. 1e), we focused on analyzing the DEGs at S2 between Ctl- and Jakitreatments. Surprisingly, out of the 969 upregulated genes between these two conditions at S2 (Fig. 1e), only one significant GO-term was identified - negative regulation of RNA polymerase II promoter activity (Additional file 4). On the contrary, GO-analysis to downregulated genes between $\mathrm{Ctl}$ - and Jaki-treatments at S2 revealed significant BPs that fall into five categories: cell cycle and DNA replication, meiotic cell cycle and spermatogenesis, DNA damage response and repair, regulation of gene expression, and stem cell maintenance (Fig. 3a, Additional file 4). Interestingly, the first 
a

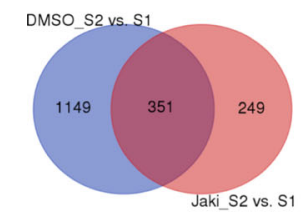

S2 vs. S1_Upregulated DEGs

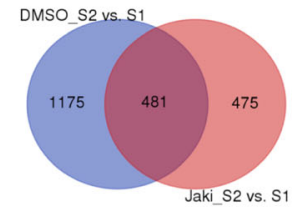

S2 vs. S1_Downregulated DEGs b
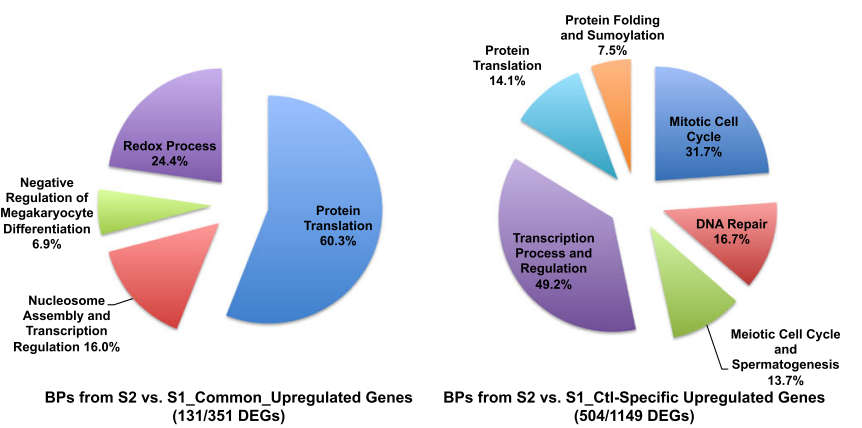
(131/351 DEGs)

C
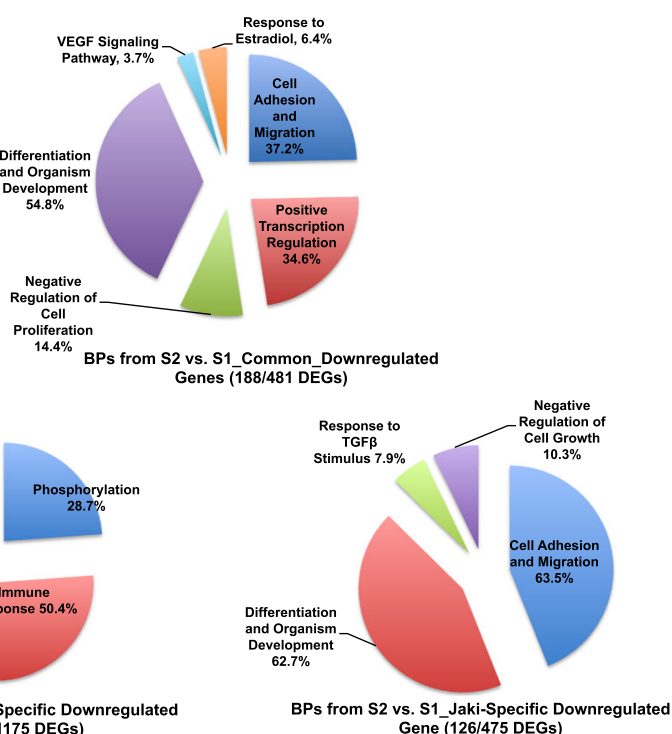

Fig. 2 JAK/STAT3 Regulates Specific Biological Events in Reprogramming. a Venn Diagrams for common or specific up- (left) or down- (right) regulated DEGs between $\mathrm{S} 1$ and $\mathrm{S} 2$ under either DMSO or Jaki condition. $\mathbf{b}$ Pie charts for summarized GO-terms using DEGs upregulated commonly between S2 and S1 (left), or upregulated in Ctl reprogramming only (Ctl-specific) (right). The number of DEGs with GO terms vs. the total number of DEGs under each comparison was shown under each chart. c Pie charts for summarized GO-terms using DEGs downregulated commonly between S2 and S1 (top), downregulated in Ctl reprogramming only (lower left), or in Jaki-specific (lower right) condition. The number of DEGs with GO terms vs. the total number of DEGs under each comparison was shown under each chart

three categories of events downregulated here were also upregulated from S1 to S2 in Ctl reprogramming (Fig. 2b-right). We wondered whether this indicates an up- or down-regulation of the same group of genes during the Ctl S1 to S2 reprogramming or in Jaki- vs. Ctl-treatment at S2, respectively. In fact, comparing the DEGs listed in each category revealed a significant portion of overlapped genes upregulated from S1 to S2 in Ctl reprogramming but downregulated by Jaki-treatment at $\mathrm{S} 2$ (Fig. 3b, Additional file 5). For example, out of the 69 meiosis and spermatogenesisrelevant genes upregulated in Ctl reprogramming, 29 (such as Text19.1, Mael, and Syce1/2 [42-45]) were downregulated at S2 by Jaki-treatment (Fig. 3b-upper left, Table 1). Similar cases were found for the genes regulating mitosis (34 out 160, such as Aurka, Cdc6, and
Ccne1 [46, 47]), and DNA damage response and repair process (27 out of 84, such as Rad51c, Mcm10, and Brca2 [48-50]), which were upregulated in Ctl reprogramming from $\mathrm{S} 1$ to S2, but downregulated by Jaki-treatment at S2 (Fig. 3b-upper right and bottom, Table 1). In addition, our RNA-seq analysis identified 35 and 130 genes at S1 and S2, respectively, which were detectable exclusively in Ctl reprogramming but absent under Jaki-treatment (Fig. 3c). GO analysis of these two groups of genes also identified similar biological events including meiosis, spermatogenesis, and oogenesis (Fig. 3c, Additional file 6). Some of these JAK/STAT3-dependent genes identified such as Stra8, Mael, and Sohlh2 are essential for the proper differentiation of germline stem cells (GSCs) both in drosophila and mammals [51-54]. 


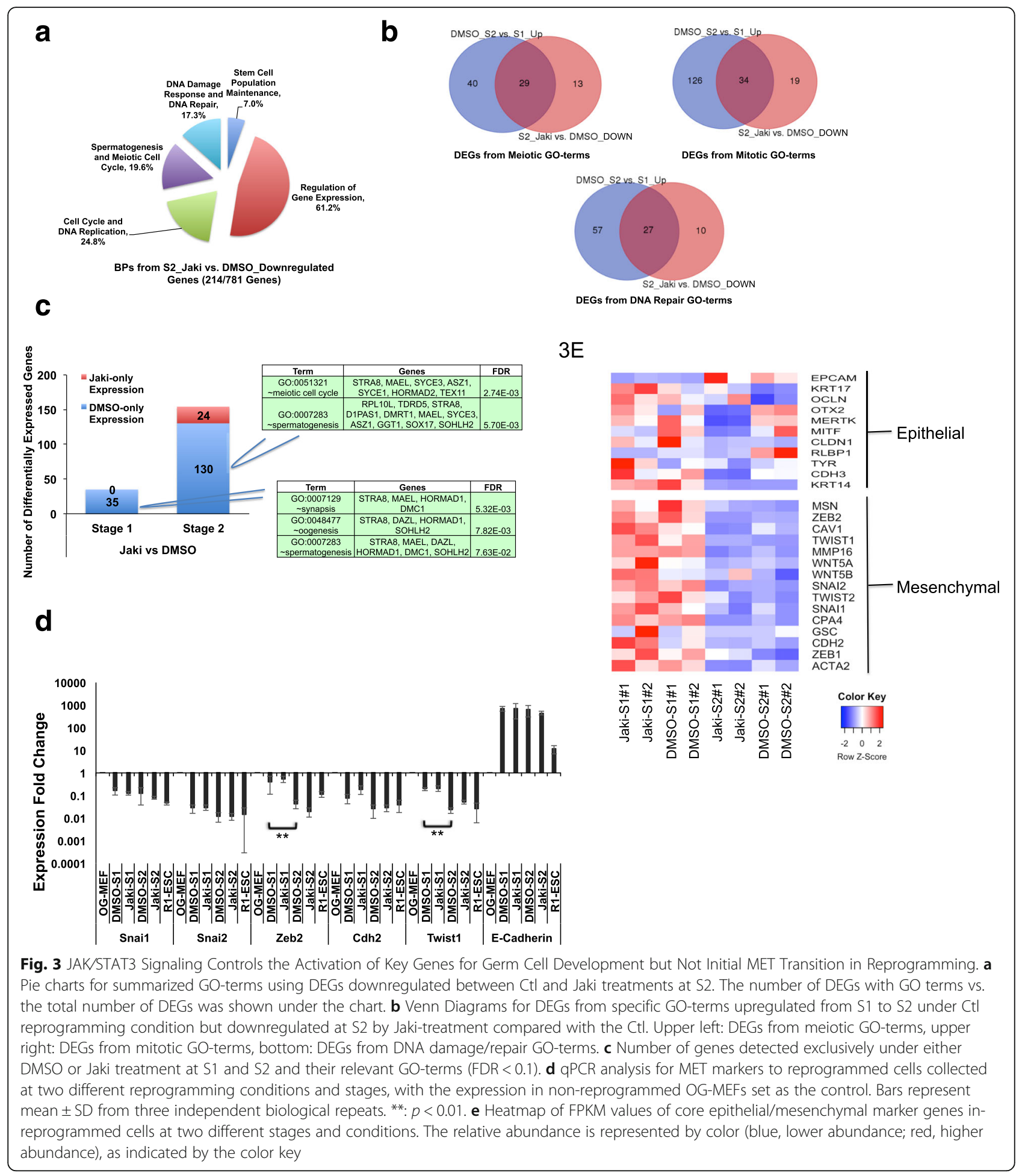

Previous study in mouse ESCs using chromatin immunoprecipitation followed by massively parallel sequencing (ChIP-seq) has identified thousands of gene loci directly bound by STAT3 [55]. We therefore asked whether the JAK/STAT3-dependent genes in reprogramming are directly targeted by STAT3, by comparing our data with the processed STAT3 ChIP-seq data [55], and with some additional STAT3 targets from re-analysis [35]. We found that more than $1 / 3$ of the JAK/STAT3dependent spermatogenesis/DNA repair genes upregulated in reprogramming are bound by STAT3, such as Brca2, Mael, Dmrt1, Chek2, etc., so is the case for 
Table 1 Common Genes from Three Categories of Biological Processes Upregulated from S1 to S2 in Ctl Reprogramming but Downregulated at $\mathrm{S} 2$ by Jaki-Treatment Compared with the DMSO Ctl

\begin{tabular}{|c|c|c|}
\hline \multicolumn{3}{|l|}{ GO-Biological Processes } \\
\hline $\begin{array}{l}\text { Spermatogenesis and } \\
\text { Meiotic Cell Cycle }\end{array}$ & $\begin{array}{l}\text { Mitotic Cell } \\
\text { Cycle }\end{array}$ & $\begin{array}{l}\text { DNA Damage } \\
\text { and Repair }\end{array}$ \\
\hline Aurka & Aurka & Ash21 \\
\hline Brca2 & $B / m$ & $B / m$ \\
\hline Ccnbl & Brca2 & Brca2 \\
\hline D1Pas1 & Bub1 & Cdc5I \\
\hline Dmrt1 & Ccnb1 & Chaf1b \\
\hline Dnmt3a & Cone1 & Chek2 \\
\hline Dnmt3l & Conf & Dna2 \\
\hline Herc4 & Cdc5I & Eef1e1 \\
\hline Hist1h1t & $C d c 6$ & Fanci \\
\hline Hsf2 & Chaf1b & Fancm \\
\hline$H s f 2 b p$ & Chek2 & Mael \\
\hline Mael & Dna2 & Mcm 10 \\
\hline Rad51c & Esco2 & Radi7 \\
\hline Rp/10l & Fanci & Rad51c \\
\hline Setx & $\operatorname{lng} 5$ & Rnf138 \\
\hline Sirt1 & Kif20b & Setx \\
\hline Sohlh2 & Kif $2 c$ & Sgk1 \\
\hline Sox17 & Mcm10 & Sirt1 \\
\hline Sycel & Mcm2 & Smarcad1 \\
\hline Syce2 & Mcm4 & Tex15 \\
\hline Sycp 1 & Mybl2 & Ticrr \\
\hline Tcfl5 & Nasp & Tipin \\
\hline Tdrd12 & Nol8 & Trim28 \\
\hline Tex11 & Nup37 & Ube2t \\
\hline Tex15 & Orc6 & Ung \\
\hline Tex19.1 & Rad17 & Usp28 \\
\hline Tex40 & Ska1 & Usp7 \\
\hline Tyro3 & Spc25 & - \\
\hline Ubaly & Ssbp 1 & - \\
\hline- & Sycel & - \\
\hline- & Syce2 & - \\
\hline- & Sycp 1 & - \\
\hline- & Ticrr & - \\
\hline- & Tipin & - \\
\hline
\end{tabular}

GO Gene Ontology Analysis. The STAT3 Direct Targets Are Marked with bold

nearly $1 / 3$ of the upregulated mitotic cell cycleassociated genes such as Ccne1, Mybl2, Cdc6, etc. (Table 1). Taken together, these data strongly indicate a specific role by JAK/STAT3 to activate genes regulating gametogenesis, meiotic, and mitotic cell cycle events in reprogramming.
JAK/STAT3 activity does not affect mesenchymal to epithelial transition in reprogramming

Blocking the MET process during reprogramming inhibits the induction of SSEA-1+ or Oct4-GFP+ colonies $[2,3]$. Interestingly, it has been shown that in carcinogenesis STAT3 stimulates epithelial to mesenchymal transition, an opposite process of MET, by upregulating key mesenchymal genes Snai1, Snai2, and Twist [56]. We wondered whether blocking STAT3 signaling might negatively impact the MET progress in reprogramming. Quantitative PCR (qPCR) analysis for MET marker genes revealed that compared to nonreprogrammed OG-MEFs, both S1 and S2 cells showed significant downregulation of mesenchymal markers including Snai1, Snai2, Cdh2, Twist1, and drastic upregulation of epithelial marker E-cadherin/Cdh1(Fig. 3d). However, Jaki-treatment at either stage had no obvious effect on the expression of these genes (Fig. 3d). This indicates a successful MET transition in reprogramming regardless of disturbed JAK/STAT3 activity. However, two mesenchymal markers (Zeb2 and Twsit1) in Ctl reprogramming condition were further downregulated at S2 than at S1 (Fig. 3d). We then explored the reported core mesenchymal and epithelial genes $[57,58]$ detected in our RNA-seq. We found that many of these mesenchyme-associated genes were downregulated from $\mathrm{S} 1$ to $\mathrm{S} 2$ in both $\mathrm{Ctl}$ and Jaki-treatment (Fig. 3e). The expression changes of core epithelial genes from S1 to S2 are more complicated, with some epithelial markers upregulated from S1 to S2 in Ctl reprogramming (such as Epcam and Rlbp1), while some others (such as Krt14, -17 , and Ocln) downregulated (Fig. 3e). These are in agreement with the previous reports that activation of Epcam is a marker for complete pluripotency at latereprogramming stage [4], whereas both Krt14 and -17 are highly expressed at intermediate-stage but downregulated at late-reprogramming stage [9]. Blocking JAK/ STAT3 activity resulted in downregulation of some epithelial markers at S2 including Otx2, Mertk, Mift, and Rlbp1, compared with the Ctl (Fig. 3e). Thus, these data show that JAK/STAT3 activity does not negatively impact the initial MET process in reprogramming. On the contrary, it stimulates the expression of some epithelial markers at late-stage reprogramming. In addition, our data also indicate that the expression of many core mesenchymal genes is further downregulated in latereprogramming stage (Fig. 3d, e). This may be important for the stabilization of the reprogrammed iPSC state.

JAK/STAT3 signaling regulates proper activation of the DIk1-Dio3 imprinted region and key pluripotent genes

The activation of maternally expressed lincRNA cluster Gtl2-Rian-Mirg in the Dlk1-Dio3 imprinted region is essential for full pluripotency establishment [15-17] 
(Additional file 1). We wondered whether JAK/STAT3 regulates the imprinting of the Dlk1-Dio3 region and Gtl2-Rian-Mirg lincRNA expression. 60 lincRNAs with significant expression changes were identified by our RNA-seq analysis (Table 2). We found both Gtt2 (also known as Meg3) and Mirg are among the 25 lincRNAs downregulated at S2 in Jaki-treatment compared with the Ctl (Fig. 4a, Table 2). qPCR analysis confirmed this finding and further revealed that all three maternally expressed lincRNAs in the Dlk1-Dio3 region were indeed downregulated at S2 by Jaki-treatment (Fig. 4b). Interestingly, examining the STAT3 ChIP-seq data also revealed the Gtl2/Meg3 gene as a direct target of STAT3 $[35,55]$ (Table 2).

The pluripotent factor Dppa3 is indispensable for proper imprinting of the Dlk1-Dio3 region in reprogramming, through antagonizing hypermethylation of IG-DMR by Dnmt3s [19]. We wondered whether JAK/ STAT3 activity regulates Dppa3 expression in reprogramming. Out of the genes significantly downregulated at S2 in Jaki-treatment compared with the Ctl, we identified some key pluripotent genes, such as Nanog, Prdm14, Sall4, Tbx3, Tet1, Tfcp2l1, and miR92-2, which belongs to the pluripotent miRNA cluster 106a-363 [59-61] (Fig. 4c, Additional file 4). Interestingly, RNAseq revealed that Dppa $3 \mathrm{mRNA}$ is only detectable in Ctl reprogramming but not in Jaki-treatment at S2 (Fig. 4c). We also found that two other Dppa family genes (Dppa2 and Dppa $5 a$ ) were significantly downregulated by Jakitreatment (Fig. 4c). qPCR analysis confirmed that while the expression of Dapp2, $-3,-4$, and $-5 a$ were all upregulated in $\mathrm{Ctl}$ reprogramming, their expression were significantly inhibited by Jaki-treatment (Fig. 4d). This correlates well with the previous studies $[35,55]$ showing that Dppa3, along with 22 out of the 34 JAK/ STAT3-depdendent pluripotent genes identified in Fig. 4c and d, are direct STAT3 targets (Fig. 4e). Furthermore, among the 34 JAK/STAT3-depdendent pluripotent genes,

Table 2 lincRNAs with Significant Expression Changes as Determined by RNA-seq Analysis

\begin{tabular}{|c|c|c|c|c|c|c|c|}
\hline \multicolumn{2}{|c|}{ Jaki-S2 vs. DMSO-S2 } & \multicolumn{2}{|c|}{ Jaki-S1 vs. DMSO-S1 } & \multicolumn{2}{|c|}{ DMSO-S2 vs. DMSO-S1 } & \multicolumn{2}{|l|}{ Jaki-S2 vs. Jaki-S1 } \\
\hline Up & Down & $\overline{U p}$ & Down & Up & Down & Up & Down \\
\hline B230217012Rik & H19 & - & H19 & 2310031A07Rik & H19 & 2810429104Rik & H19 \\
\hline Cep83os & 1700001L05Rik & - & Gm26809 & 2810429104Rik & 1500026H17Rik & 4732463B04Rik & 3300005D01Rik \\
\hline Gm15675 & 1700018B24Rik & - & Lncencl & Epb4114aos & 1600025M17Rik & 4930509G22Rik & 6330403K07Rik \\
\hline Gm20732 & 1700019E08Rik & - & Platr20 & EU599041 & 2610035D17Rik & Gm19705 & $9030622 \mathrm{O} 22 \mathrm{Rik}$ \\
\hline Gm26569 & 1700057H21Rik & - & Platr4 & Gm17275 & 4930431F12Rik & Gm807 & Gm16211 \\
\hline Gm807 & 2210417A02Rik & - & - & Gm27010 & Gm10660 & - & Gm26809 \\
\hline Mirt1 & 2410018L13Rik & - & - & Gm7976 & Gm11033 & - & Gm26905 \\
\hline Neat1 & 4930544I03Rik & - & - & Kis2 & Gm15298 & - & $\operatorname{Meg} 3$ \\
\hline- & 4930591A17Rik & - & - & Lncencl & Gm15675 & - & Neat1 \\
\hline- & EU599041 & - & - & Mir17hg & Gm26809 & - & - \\
\hline- & Gm12688 & - & - & Mirg & Gm26981 & - & - \\
\hline- & Gm26579 & - & - & Platr25 & Gm42418 & - & - \\
\hline- & Gm26635 & - & - & Platr4 & Malat1 & - & - \\
\hline- & Gm26639 & - & - & Platr7 & Mirt1 & - & - \\
\hline- & Gm26715 & - & - & - & Neat1 & - & - \\
\hline - & Gm26905 & - & - & - & Otx2os1 & - & - \\
\hline- & Gm2694 & - & - & - & - & - & - \\
\hline- & Gm4425 & - & - & - & - & - & - \\
\hline- & Gm7976 & - & - & - & - & - & - \\
\hline- & Lncencl & - & - & - & - & - & - \\
\hline- & Meg3 & - & - & - & - & - & - \\
\hline- & Mirg & - & - & - & - & - & - \\
\hline- & Platr25 & - & - & - & - & - & - \\
\hline- & Platr4 & - & - & - & - & - & - \\
\hline - & Platr7 & - & - & - & - & - & - \\
\hline
\end{tabular}




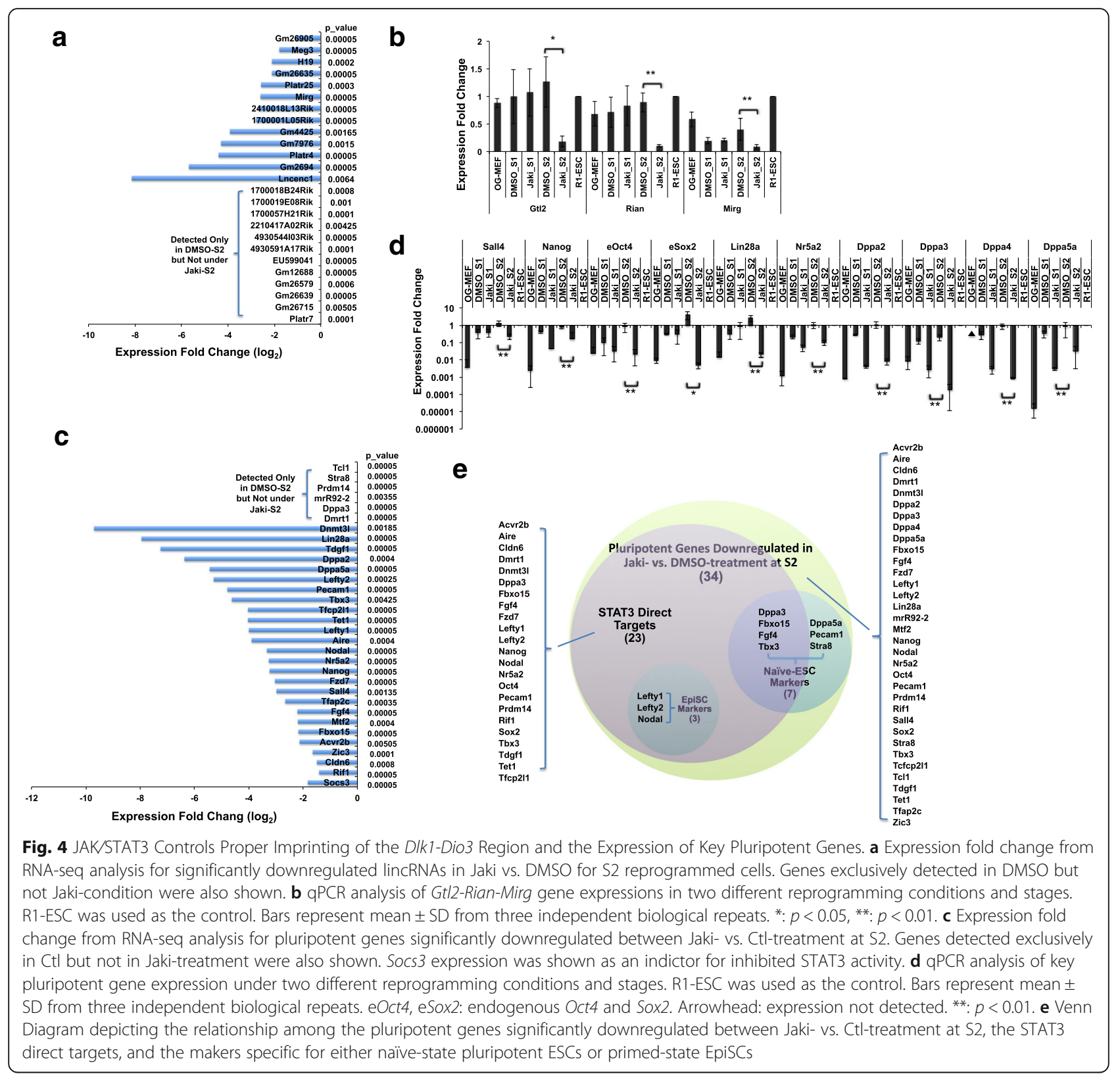

7 (including the germline markers Dppa3 and Stra8) are naïve-state ESC-specific $[62,63]$, with 4 out of these 7 genes (Dppa3, Fbxo15, Fgf4, and Tbx3) being direct targets of STAT3 (Fig. 4e). Interestingly, we also found that the expression of 3 primed-state EpiSC markers (Nodal, Lefty1, and Lefty2) $[62,63]$ is JAK/STAT3-dependent (Fig. 4c), with these gene loci bound by STAT3 [55] (Fig. 4e). This is consistent with the previous studies showing both LIF and ACTIVIN/NODAL promote the propagation of naïve-state ESCs, while NODAL signal does not affect naïve ESC pluripotency in serum-free condition $[64,65]$. Thus, the proper expression of Gtl2-Rian-Mirg lincRNAs is regulated by JAK/STAT3 at late-reprogramming stage, and this may be achieved through direct STAT3 binding to Gtl2/Meg3 and through JAK/STAT3-dependent Dppa3 activation in reprogramming. In the meanwhile, JAK/ STAT3 promotes complete pluripotency establishment by stimulating the activation of key pluripotent genes, including the naïve-state and germ cell specific markers.

\section{JAK/STAT3 regulates expression of key histone modifiers during reprogramming}

Epigenetic changes during reprogramming are essential to activate core pluripotent genes, and silence transgenes and lineage commitment genes $[4,6,8,9]$. We previously identified that JAK/STAT3 activates the expression 
of de novo DNA methyltransferase Dnmt3a, - 3b, $-3 l$, and suppresses the histone deacetylases (Hdacs) expression [29]. Our RNA-seq data agree with these findings and further revealed an increased Hdac10 expression in S2 in the presence of Jaki (Fig. 5a). In addition, we discovered an increased expression of histone/lysine acetyltransferases (Hats/Kats) including Hat1, Kat5, -6b, and - 8 [66] from S1 to S2 in Ctl reprogramming (Fig. 5a). Blocking JAK/STAT3 activity, however, downregulated Hats/Kats including Hat1, Kat6b, and Ncoa3 [67, 68] at S2 compared with the Ctl (Fig. 5a). A direct binding to Hdac10, Hat1, and Ncoa3 gene loci in ESCs by STAT3 was also shown before [55]. These data thus indicate that in addition to inhibition of Hdacs, JAK/STAT3 also selectively stimulates Hats/Kats expression to promote histone acetylation in reprogramming.

PRC2 mediated H3K27 trimethylation is necessary to suppress core developmental genes for successful reprogramming [69]. Moreover, in ESCs, PRC2 antagonizes hypermethylation of the Dlk1-Dio3 IG-DMR region by de novo Dnmt3s, and depletion of PRC2 components Eed, Jarid2, or the major methyltransferase Ezh2 suppressed maternal Gtl2-Rian-Mirg expression, due to hypermethylation of IG-DMR [20]. As we have observed that JAK/STAT3 promotes the expression of both Gtl2Rian-Mirg lincRNAs and de novo Dnmts at S2 (Figs. 4b, $5 \mathrm{a}$, and reference [29]), we wondered if JAK/STAT3 would also regulate PRC2 activity to ensure proper imprinting of the Dlk1-Dio3 region. In fact, RNA-seq data and qPCR analyses revealed that the expression of most PRC2 components including Eed, Rbbp4, Jarid2, Mtf2, esPRC2p48, Suz12, and Ezh2 increased from S1 to S2 in Ctl reprogramming (Fig. 5b, c). However, blocking JAK STAT3 significantly inhibited the expression of these PRC2 components (except for Rbbp4 and Suz12) at S2 compared to the Ctl (Fig. 5b, c). On the other hand, the expression of another PRC2 methyltransferases - Ezh1 decreased from S1 to S2 in Ctl reprogramming, and Jaki-treatment inhibited this decrease (Fig. 5c). Interestingly, it was also reported that inhibiting Ezh1 in a

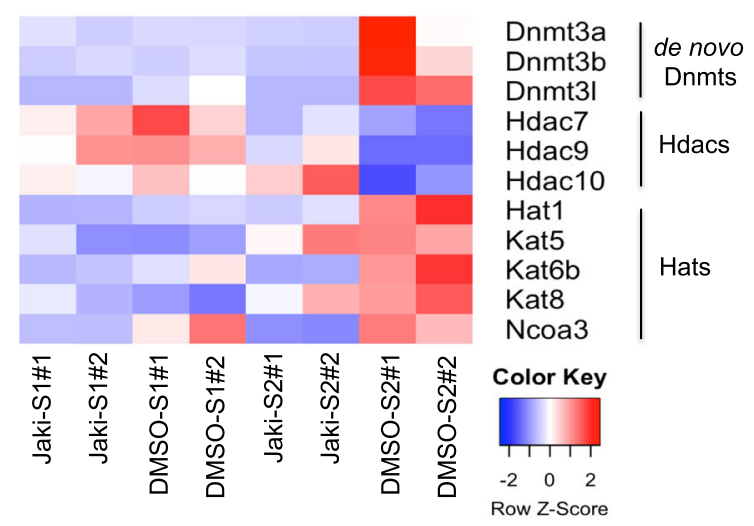

\section{b}

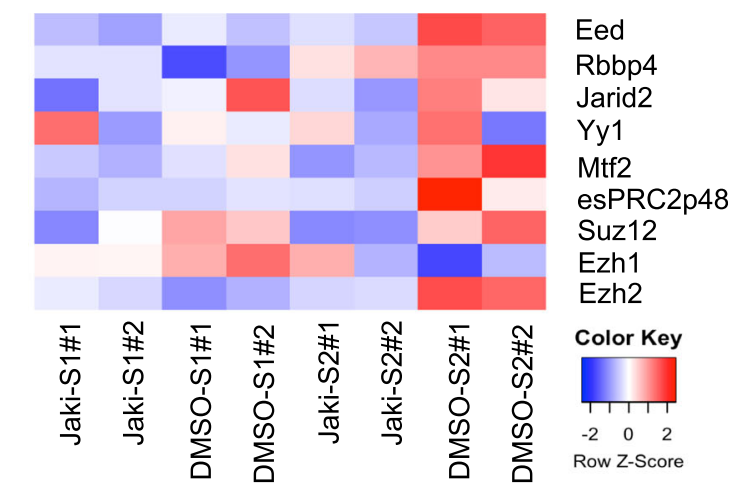

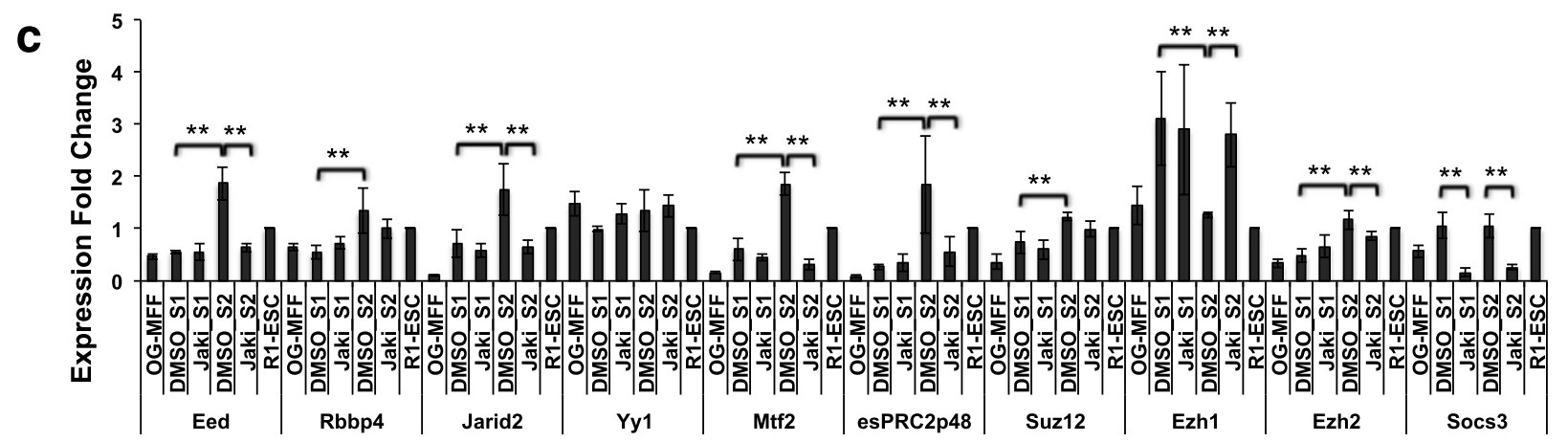

Fig. 5 JAK/STAT3 Controls the Expression of Key Histone Modifiers. a Heatmap of FPKM value comparison for Dnmt, Hdac, and Hat/Kat genes detected under two different treatments and reprogramming stages. The relative abundance is represented by color (blue, lower abundance; red, higher abundance), as indicated by the color key. b Heatmap of FPKM value comparison for PRC2 component genes detected under two different treatments and reprogramming stages. The relative abundance is represented by color (blue, lower abundance; red, higher abundance), as indicated by the color key. c qPCR analysis of PRC2 component gene expressions in two different reprogramming conditions and stages. R1-ESC was used as the control. Bars represent mean \pm SD from three independent experiments. **: $p<0.01$ 
reprogramming stimulated human iPSC generation [8]. Previous STAT3 ChIP-seq analysis again revealed that Eed, Ezh2, Jarid2, and Rbbp4 are direct targets of STAT3 $[35,55]$. Taken together, these data strongly argue that JAK/STAT3 stimulates PCR2 activity in latereprogramming stage, which correlates with the proper expression of Gtl2-Rian-Mirg lincRNAs, an essential event for complete pluripotency establishment.

\section{JAK/STAT3 activity is crucial for activating pluripotent DNA loci during reprogramming}

To test the epigenetic modulation of pluripotent loci including Oct4, Nanog, and the Dlk1-Dio3 region by JAK STAT3, we employed the Jaki-treated pre-iPSCs reprogrammed from OG-MEFs and collected at S2. These cells could be passaged continuously in the presence of Jaki or a LIF-neutralizing antibody (LIFAb) and remained largely GFP-, thus further validating the specificity of Jaki on inhibiting LIF/STAT3 signaling (Fig. 6a). We asked whether removing the inhibition of JAK/ STAT3 could resume the halted reprogramming process. Removing Jaki from the culture medium (LIF+ condition) led to a gradual conversion of GFP- colonies to GFP+ in 3 weeks, while those colonies left in either Jaki or LIFAb treatment remained GFP-, as confirmed by fluorescence activated cell sorting (FACS) (Fig. 6a, b). Previously we reported that these GFP- pre-iPSCs had hypermethylated Oct4 and Nanog promoters [29]. Our study here further revealed that the expressions of some important genes responsible for DNA-demethylation in reprogramming are JAK/STAT3-dependent, including the DNA hydroxylase Tet1 that promotes Oct4 demethylation and activation [70, 71], and Dppa3 and PRC2 genes that prevent de novo methylation of Dlk1-Dio3 region $[19,69]$ (Figs. 4c-e, 5b, c). We therefore asked whether removing JAK/STAT3 inhibition is necessary to re-activate these loci. We extracted DNAs from the FACS sorted cells and analyzed their methylation status using bisulfite sequencing. We found that upon restoring JAK/STAT3 signaling, the Oct4 and Nanog promoter loci were completely demethylated in GFP+ cells, whereas in cells kept in Jaki or LIFAb these regions still remained hypermethylated (Fig. 6c, Additional file 7). Interestingly, the GFP- cells under LIF-only condition showed a partial demethylation for the Oct4 promoter (Fig. 6c), indicating resumed but still incomplete reprogramming status in these cells. Importantly, restoring JAK/STAT3 activity also led to decreased methylation of IG-DMR in the GFP+ cells, while the cells kept in Jakior LIFAb-treatment still remained hypermethylated for this region (Fig. 6d). Thus, JAK/STAT3 signaling is indeed a prerequisite for activation of key pluripotent genomic loci and the Dlk1-Dio3 region in reprogramming by promoting their DNA demethylation.
Previously we discovered an epigenetic role by JAK/ STAT3 for pluripotency establishment in reprogramming, through regulation of Dnmts and Hdacs [29]. Based on our study here, we propose an updated model for JAK/STAT3 regulated naïve-state pluripotency establishment at late-stage reprogramming (Fig. 5d), where JAK/STAT3-dependent stimulation of Tet1, Dppa3, PRC2, and Hats/Kats expression and inhibition of Hdacs expression promote the euchromatic state at pluripotent loci such as Oct4, Nanog, and Dlk1-Dio3 for their full activation by OKSM and other pluripotent factors including STAT3, while JAK/STAT3-dependent de novo Dnmt3s expression helps silence commitment genes and OKSM transgenes. These, together with the JAK/ STAT3-stimulated activation of germ cell-specific genes, promote the establishment of ground state, germline transmission-capable naïve-pluripotency in reprogramming.

\section{Discussion}

The LIF regulated JAK/STAT3 pathway is important for naïve-state pluripotency establishment across species for iPSC generation [72]. Although many downstream targets of STAT3 have been reported, the complete understanding of JAK/STAT3 mediated pluripotency establishment has not been achieved. We performed RNA-seq to analyze JAK/STAT3 mediated reprogramming and identified biological events and DEGs specifically regulated by JAK/STAT3 activity during the iPSC induction process. We found that during late-stage reprogramming, JAK/STAT3 signaling regulates gametogenesis events especially the spermatogenesis, mitotic/ meiotic cell cycle, and the DNA damage and repair process - an essential process to ensure DNA integrity during mitotic/meiotic cell division [73]. It is well established that in Drosophila, JAK/STAT activity is required to for GSC maintenance in both testis and ovary [7477]. However, an understanding of the role by JAK/ STAT in GSC regulation in mammals is still limited [78, 79]. Our analysis revealed that JAK/STAT3 regulates the expression of key germ cell developmental genes such as Text19.1, Stra8, Mael, Sohlh2, Syce1/2, etc. In addition, we also identified that JAK/STAT3 stimulates the expression of pluripotent transcription factors such as Prdm14 and Dmrt1, which are critical for gonad development and GSC specification in mammals [80, 81]. Naïve-state pluripotency is important for the chimera and germline chimera formation capability of mouse and human ESCs/iPSCs [62, 63, 82-84]. The JAK/STAT3 regulated gametogenesis/meiotic events in reprogramming could be crucial for this germline chimerism capacity establishment. Our study provides valuable mechanistic insight and information database for further 


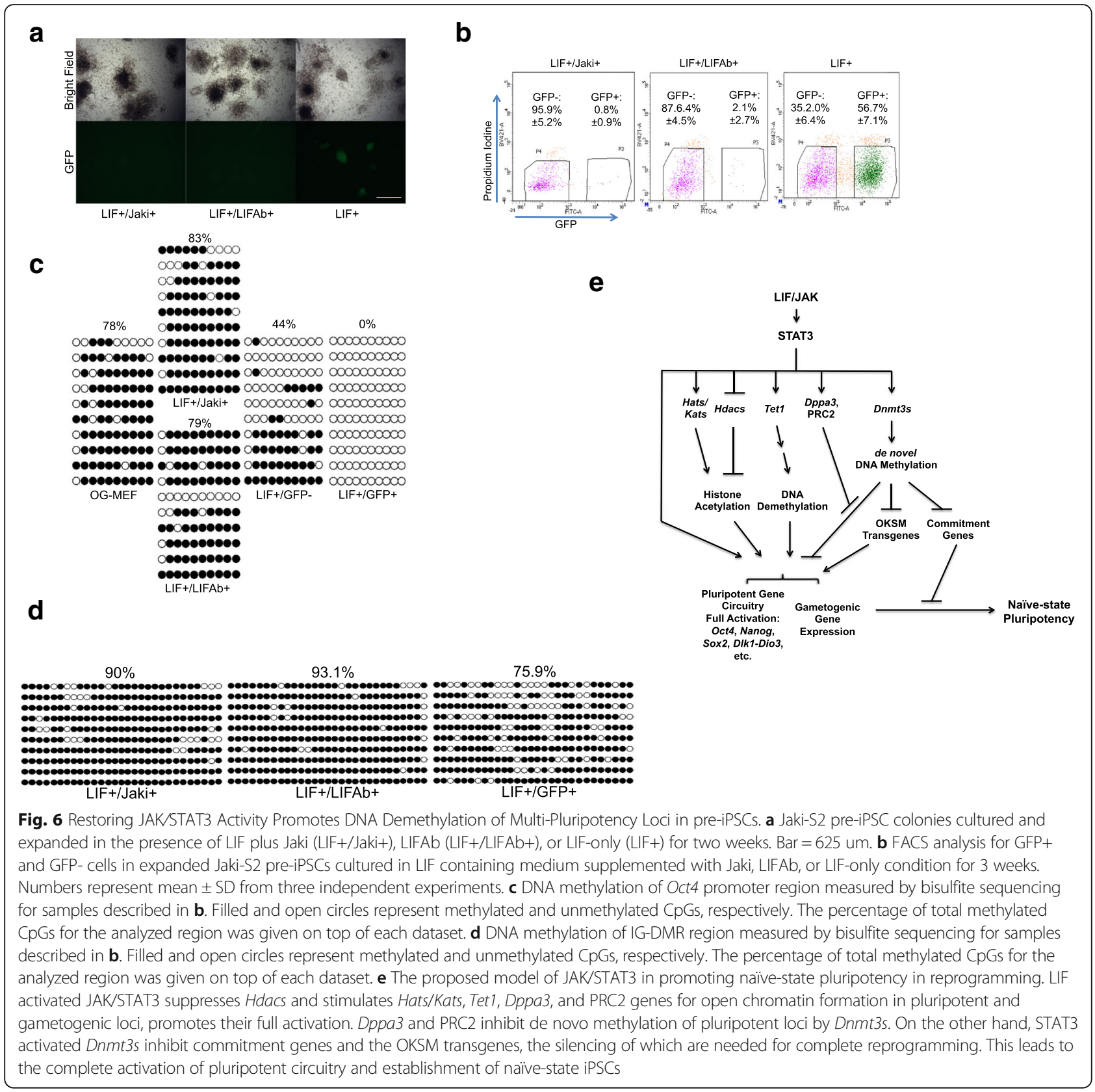

elucidation of reprogramming as well as meiotic processes specifically regulated by JAK/STAT3 signaling.

We discovered that JAK/STAT3 plays a critical role to regulate the Dlk1-Dio3 imprinted region. Activation of the maternally expressed Gtl2-Rian-Mirg lincRNAs in this region serves as a key event for pluripotency establishment in late-stage reprogramming [15]. Recently it was shown that Dppa3 is expressed only in iPSCs capable of chimera-formation and specifically blocks the Dnmt3a mediated methylation of IG-DMR [19]. One of the mechanisms therefore JAK/STAT3 regulates imprinting of the Dlk1-Dio3 region might be via JAK/
STAT3-dependent stimulation of Dppa3 expression. However, other mechanisms may also play a role here. The PRC2 components Ezh2, Eed, and Jarid2 were reported to prevent Dnmt3s from methylating the Dlk1Dio3 region, thus maintaining the expression of maternal Gtl2-Rian-Mirg in mouse ESCs [20]. We found that blocking JAK/STAT3 activity inhibits the expression of many PRC2 components. Thus JAK/STAT3 may also regulate proper imprinting of the Dlk1-Dio3 region through stimulating PRC2 activity. In accordance with these findings, we discovered that removing Jaki in the LIF+ medium promotes demethylation of IG-DMR, the 
region controlling Gtl2-Rian-Mirg expression. In addition, histone acetylation of the Dlk1-Dio3 region correlates with the activity of this region, and inhibiting Hdacs activates the expression of Gtl2-Rian-Mirg in reprogramming [16]. We previously reported that JAK/ STAT3 activity downregulates the expression of Hdacs during reprogramming [29] and here we further discovered that in addition to Hdacs, inhibition of JAK/STAT3 also blocks the expression of certain Hats/Kats. How exactly JAK/STAT3 activity regulates the proper imprinting of the Dlk1-Dio3 region is certainly of high interest and warrants future investigation.

We further identified that JAK/STAT3 activity regulates the activation of a number of key pluripotent factors such as the Dppa family genes. The expression of Dppa3 is present only in iPSCs with chimera-forming capacity, and blocking its expression results in the generation of pre-iPSCs only [19]. One possible mechanism that JAK/STAT3 may regulate the expression of Dppa3 can be through promoting DNA demethylation at its regulatory sequence, similar as what we demonstrated here that JAK/STAT3 activity is essential for the demethylation of Oct4 and Nanog promoters in reprogramming. Additionally, one of the downregulated pluripotent genes by Jaki-treatment - $T b x 3$ has been reported to prevent ESC differentiation through promoting the expression of Dppa3 [85]. Exactly how JAK/STAT3 signaling activates these pluripotent genes to promote complete reprogramming is currently under active investigation. Nevertheless our results correlate well with the previous ChIP-seq analyses $[35,55]$ and strongly indicate that an activated STAT3 elicits layers of regulatory mechanisms over its targets, either directly or through control of specific epigenetic modulators. Our analysis demonstrates that JAK/STAT3 orchestrates events at later-reprogramming stage from upregulation of gametogenesis, meiotic/mitotic, and DNA damage/repair processes, to stimulation of key pluripotent genes and epigenetic regulators for complete pluripotency establishment.

\section{Conclusions}

We performed transcriptome analysis to investigate the genomic expression dynamics regulated by JAK/STAT3 activity during somatic cell reprogramming. We describe JAK/STAT3-specific upregulation of biological events such as gametogenesis and cell cycle processes in reprogrammed cells. We found that JAK/STAT3 does not affect MET transition in reprogramming but regulates the expression of some core mesenchymal/epithelial markers, and describe key pluripotent transcription factors, epigenetic modulators, and non-coding RNAs regulated by JAK/STAT3. We show that JAK/STAT3 activity is necessary for proper imprinting of the Dlk1-Dio3 region, which is associated with the JAK/STAT3dependent stimulation of Dppa3 and PRC2 components in reprogramming. We further demonstrate that JAK/ STAT3 activity is essential for promoting DNA demethylation of pluripotent loci including Oct4, Nanog, and Dlk1-Dio3 regions at late-reprogramming stage. Our data elucidate new mechanisms for JAK/STAT3 promoted pluripotency establishment in reprogramming, which are valuable for improving the generation of naïve-state iPSCs across species.

\section{Methods}

\section{Chemicals and recombinant DNA constructs}

The Jak inhibitor I (Jaki) was from EMD (Billierica, MA, USA). LIF antibody (LIFAb) was from Santa Cruz (Santa Crutz, CA, USA). Retroviral pMXs-mouse Oct4, Klf4, Sox2, and $c-M y c$ were from Addgene (Cambridge, MA, USA). All constructs were verified by DNA sequencing.

\section{RNA isolation, library construction and sequencing}

Total RNA was isolated from reprogrammed cells with different treatments using RNeasy Mini kit (Qiagen, Valencia, CA) and reverse transcribed using the SuperScript III Reverse Transcription Kit (Invitrogen, Grand Island, NY). The quality of Total RNA was examined with the Aglient RNA 1000 Nano kit (Aglient Technologies, Santa Clara, CA). rRNA was then removed by using Ribo-Zero-rRNA Removal kit (Epicentre, Madison, WI). $500 \mathrm{ng}$ of rRNA-depleted total RNA from each sample was used to prepare the RNA sequencing library following the manufacturer's instructions by SOLiD Total RNA-seq Kit (Life Technologies, Grand Island, NY). Finally, sequencing libraries were quantified by using Agilent 2100 bioanalyzer and then barcoded, multiplexed, and sequenced on a 5500xl Genetic Analyzer at the Center for Applied Genetics and Technology, University of Connecticut. We obtained approximately 240 million sequencing reads with a read length of 75-bp from 8 samples. The raw FASTQ files and normalized gene expression levels are available at Gene Expression Omnibus (GEO) (www.ncbi.nlm.nih.gov/geo) under the accession number GSE97261.

\section{Data processing of RNA-seq}

Sequencing reads were trimmed as previously described [86]. Briefly, sequencing adapters were trimmed using Cutadapt and low quality reads were pre-filtered by FASTX-Toolkit before mapping. The quality of reads after filtering was examined using fastQC. For mapping, mouse genomic sequence and RefSeq gene coordinate (GRCm38/mm10) were downloaded from the UCSC genome browser. All filtered reads were aligned to mouse reference genome by Tophat (v2.0.10) using SAMtools (v0.1.18) AND Bowtie (v2.1.0) with default 
parameters [87, 88]. Individual mapped reads were fed to Cufflinks (v1.2.1) [88] to contruct transcriptome models and any novel genes and transcripts that did not fit the supplied gene models were also assembled. Cuffmerge [88] was used to converge individual transcriptome to produce a master gene model. Then Cufflinks was run to calculate Fragments Per Kilobase of exon model per Million mapped fragments (FPKM) by using RefSeq genes as reference [88]. A matrix of Pearson correlation coefficient was created using $\mathrm{R}$ Package, which was in turn used to create the heatmap. Differentially expressed genes between two stages were identified using default parameters in Cuffdiff [87]. We also included an additional bias detection and correction algorithm filter available in the Cufflinks package to improve the accuracy of transcript abundance estimates. Genes were deemed differentially expressed between subsequent developmental stages if they showed a FDR (adjusted $p$-value or q-value) of less than 0.05. Expression pattern clusters were generated by the K-means clustering algorithm using $R$.

\section{Gene ontology, meta-analysis, and PCA analysis}

Functional annotation enrichment analysis for Gene Ontology (GO) and pathway analyses were conducted by Database for Annotation, Visualization and Integrated Discovery Bioinformatics Resource (DAVID) [89]. We summarized all similar sub-GO terms and pathways into an overarching term, and $p$-values are shown for the representative terms. Principle component analysis (PCA) was performed using the GeneXplain platform (www.genexplain.com).

\section{qPCR analysis}

Total RNA were extracted using a RNeasy Extraction kit (Qiagen, Hilden, Germany), reverse transcribed using a iScript Reverse Transcription Kit (Bio-Rad, Hercules, CA, USA) and PCR amplified with specific primers (Primer sequences available upon request). qRT-PCR was performed using SYBR Green PCR Master Mix (BioRad) and the ABI 7500 Fast instrument, and data analyzed using the 7500 software version 2.0 .2 provided with the instrument. All values were normalized with GAPDH as the internal control and relative mRNA expressions were quantified using either MEFs or R1-ESCs as the reference, which is specified in each figure legend. Data were analyzed with One Way ANOVA or the Student's $t$-test.

\section{Bisulfite sequencing}

For bisulfite sequencing, genomic DNAs were extracted and bisulfite converted using an EpiTeck Bilsulfite Kit (Qiagen). Oct4 and Nanog promoter regions were amplified using PCR primers described previously [29]. Nested-PCR was used to clone the IG-DMR region, with the first round amplification using primer pairs: $5^{\prime}$ 'TAAGTGTTGTGGTTTGTTATGGGTA-3' (forward) and 5'-CCATCCCCTATACTCAAAACATTCT-3' (reverse), and the second round using primer pairs: 5'TACCGGACTCAGATCT TGGTTTGTTATGGGTA AGTTTTATG (forward) and 3'-GTCGACTGCAGAATTC CTTCCCTCACTCCAAAAATTAAAA (reverse), with the bold letters indicate vector sequences for fusion cloning. PCR were performed with Taq $2 \times$ Master Mix (New England Biolabs, Ipswich, MA, USA) and cloned using an In-Fusion HD Cloning System (Clontech) into pIRES2-DsRed vector digested by BglII and EcoRI (New England Biolabs). Clones were picked, cultured in $5 \mathrm{ml} \mathrm{LB}$ medium with antibiotics overnight, and plasmid DNAs were extracted using a Qiaprep Mini Kit (Qiagen) and sequenced by regular Sanger DNA sequencing.

\section{Cell culture and reprogramming assay}

Generation of Jaki-treated pre-iPSCs using retroviral transduction and reprogramming medium was described previously [29]. The reprogramming medium contains 1:1 mixture of KSR-ESC medium containing $76 \%$ KO-DMEM, 20\% KSR, 1\% 100× glutamax, $1 \%$ $100 \times$ non-essential amino acids, and $0.5 \times$ penicillin/ streptomycin (Invitrogen), and supplemented with $1 \%$ $100 \times \beta$-mercaptoethanol and $1000 \mathrm{U} / \mathrm{ml}$ LIF (Millipore, Billerica, MA, USA), and Serum-ESC medium with $76 \%$ DMEM, 20\% ESC-qualified FBS from Hyclone (Fisher Scientific, Pittsburg, PA, USA), 1\% 100× glutamax, 1\% 100× non-essential amino acids, $0.5 \times$ penicillin/streptomycin, $1 \% 100 \times \beta$-mercaptoethanol, and $1000 \mathrm{U} / \mathrm{ml} \mathrm{LIF}$. Induced colonies were further expanded in the presence of Jaki in ESC medium containing 76\% KO-DMEM, 20\% KSR, 1\% $100 \times$ glutamax, $1 \% 100 \times$ non-essential amino acids, and $0.5 \times$ penicillin/streptomycin (Invitrogen), and supplemented with $1 \% \quad 100 \times \quad \beta$-mercaptoethanol and $1000 \mathrm{U} / \mathrm{ml}$ LIF. For reprogramming assay, cells were seeded at a density of 0.25 million cells per 24-wellplate pre-seeded with mitomycin C treated CD1 MEF feeders on Day -1 . On day 0 the cells were infected with retroviral OKSM, and medium replaced after $24 \mathrm{~h}$. On day 2 the ESC medium containing $1 \mu \mathrm{M}$ Jaki or $0.5 \mu \mathrm{g} / \mathrm{mL}$ LIFAb were applied to all conditions except for the positive control wells. Media were replaced every two days. GFP-expressing colonies were scored between 2 to 3 weeks after initial viral transduction under a Nikon fluorescence microscope, or subjected to fluorescence-activated cell sorting (FACS) for the percentage of GFP+ cells at the UConn Bioservice Center. 


\section{Additional files}

Additional file 1: Schematic representation of the DIk1-Dio3 region at mouse chromosome 12qF1. The Gt/2-Rian-Mirg lincRNAs are expressed from the maternally inherited chromosome, while the protein coding DIk1, Rt 11, and Dio3 genes are expressed from the paternally inherited chromosome. IG-DMR is paternally methylated but demethylated in maternal chromosome to control expression of the Gt/2-Rian-Mirg lincRNAs. (PDF $61 \mathrm{~kb}$ )

Additional file 2: Cuffdiff Analysis Results Table of DEGs Between CtI S2 vs. S1 (DMSO_S2 vs. S1), Jaki S2 vs. S1 (Jaki_S2 vs. S1), Jaki vs. DMSO at S1 (S2_Jaki vs. DMSO), and Jaki vs. DMSO at S1 (S1_Jaki vs. DMSO). (XLSX $829 \mathrm{~kb})$

Additional file 3: DAVID Analysis Table of Biological Processes (FDR < 0.05) for Common or Specific Up- or Down-regulated DEGs Between Ct reprogramming (DMSO_S2 vs. S1) and Jaki-reprogramming (Jaki_S2 vs. S1). (XLSX 60 kb)

Additional file 4: DAVID Analysis Table of Biological Processes (FDR < 0.05) for Up- or Down-regulated DEGs Between Jaki vs. DMSO at S2. (XLSX $28 \mathrm{~kb}$ )

Additional file 5: Table for DEGs listed in Spermatogenesis/Meiotic, Mitotic, and DNA Repair GO-terms That Are Upregulated between CtI S2 vs. S1 comparison but Downregulated at $\mathrm{S} 2$ in Jaki vs. Ctl comparison. (XLSX $60 \mathrm{~kb}$ )

Additional file 6: Table for S1- or S2-specifically Expressed Genes under DMSO Ctl or Jaki-Treatment at S1 or S2. (XLSX $102 \mathrm{~kb})$

Additional file 7: JAK/STAT3 Activity Is Needed to Activate Pluripotent Loci in Reprogramming. DNA methylation of Nanog promoter region measured by bisulfite sequencing for samples described in Fig. 6b. Filled and open circles represent methylated and unmethylated CpGs, respectively. The percentage of total methylated $\mathrm{CpGs}$ for the analyzed region was given on top of each dataset. (PDF $322 \mathrm{~kb}$ )

\section{Abbreviations}

BPs: Biological processes; Ctl: Control; DEGs: Differentially expressed genes; Dnmt: DNA methyltransferase; Dppa: Developmental pluripotency associated; FACS: Fluorescence activated cell sorting; FDR: False discovery rate; FPKM: Fragments Per Kilobase of exon model per Million mapped fragments; GO: Gene ontology; H3K37: Histone 3 lysine 27; Hats/Kats: Histone/lysine acetyltransferases; IG-DMR: Intergenic differential methylated region; iPSCs: induced pluripotent stem cells; JAK/STAT3: Janus kinase/signal transducer and activator of transcription 3; Jaki: Jak inhibitor l; LIF: Leukemia inhibitory factor; LIFAb: LIF-Neutralizing antibody; lincRNAs: Long intervening non-coding RNAs; MEFs: Mouse embryonic fibroblasts; MET: Mesenchymal to epithelial transition; miRNAs: microRNAs; OG-MEFs: MEFs with GFP expression controlled by the Oct4 distal enhancer region; OKSM: Oct4, KIf4, Sox2, and cMyc; PCA: Principle component analysis; PRC2: Polycomb repressive complex 2

Acknowledgements

Not applicable

\section{Funding}

The design of the study, data collection, analysis, and interpretation, and manuscript writing were supported by the USDA National Institute of Food and Agriculture (NIFA) grants CONS-2013-03194 to Y.T. and X.T., USDA-ARS agreement 58-8042-5-047 to X.T., the USDA W2171 regional project to Y.T. and X.T., and by Agriculture and Food Research Initiative Competitive Grant no. 2016-67016-24894 from the USDA/NIFA to Y.T.

\section{Availability of data and materials}

The datasets generated and/or analyzed during the current study are available in the GEO repository under the accession number GSE97261 with the flowing link.

https://www.ncbi.nlm.nih.gov/geo/query/acc.cgi?acc=GSE97261

\section{Authors' contributions}

LW, ZJ: Collection and assembly of data, Data analysis and interpretation, Manuscript writing. DH, JD, CH: Collection and assembly of data, Data analysis and interpretation. SS, KV, YY: Collection and assembly of data. MZ, JW: Conception and design, Data analysis and interpretation. XT, YT: Conception and design, Financial support, Collection and assembly of data, Data analysis and interpretation, Manuscript writing. All authors read and approved the final manuscript.

Ethics approval and consent to participate

Not applicable

\section{Consent for publication}

Not applicable

\section{Competing interests}

The authors declare that they have no competing interests.

\section{Publisher's Note}

Springer Nature remains neutral with regard to jurisdictional claims in published maps and institutional affiliations.

\section{Author details}

${ }^{1}$ Department of Animal Science, Institute for Systems Genomics, University of Connecticut, Storrs, CT, USA. ${ }^{2}$ State Key Laboratory for Conservation and Utilization of Subtropical Agro-Bioresources, Animal Reproduction Institute, Guangxi University, Nanning, Guangxi, People's Republic of China. ${ }^{3}$ Department of Ecology and Evolutionary Biology, Computational Biology Core, Institute for Systems Genomics, University of Connecticut, Storrs, CT, USA. ${ }^{4}$ Present address: School of Animal Science, Louisiana State University, Baton Rouge, LA, USA.

Received: 23 June 2017 Accepted: 29 January 2018

Published online: 06 March 2018

\section{References}

1. Takahashi K, Yamanaka S. Induction of pluripotent stem cells from mouse embryonic and adult fibroblast cultures by defined factors. Cell. 2006;126(4): 663-76

2. Li R, Liang J, Ni S, Zhou T, Qing X, Li H, He W, Chen J, Li F, Zhuang Q, et al. A mesenchymal-to-epithelial transition initiates and is required for the nuclear reprogramming of mouse fibroblasts. Cell Stem Cell. 2010;7(1):51-63.

3. Samavarchi-Tehrani P, Golipour A, David L, Sung HK, Beyer TA, Datti A, Woltjen K, Nagy A, Wrana JL. Functional genomics reveals a BMP-driven mesenchymal-to-epithelial transition in the initiation of somatic cell reprogramming. Cell Stem Cell. 2010;7(1):64-77.

4. Polo JM, Anderssen E, Walsh RM, Schwarz BA, Nefzger CM, Lim SM, Borkent M, Apostolou E, Alaei S, Cloutier J, et al. A molecular roadmap of reprogramming somatic cells into iPS cells. Cell. 2012;151(7):1617-32.

5. Golipour A, David L, Liu Y, Jayakumaran G, Hirsch CL, Trcka D, Wrana JL. A late transition in somatic cell reprogramming requires regulators distinct from the pluripotency network. Cell Stem Cell. 2012;11(6):769-82.

6. Buganim Y, Faddah DA, Cheng AW, Itskovich E, Markoulaki S, Ganz K, Klemm SL, van Oudenaarden A, Jaenisch R. Single-cell expression analyses during cellular reprogramming reveal an early stochastic and a late hierarchic phase. Cell. 2012;150(6):1209-22.

7. Buganim Y, Faddah DA, Jaenisch R. Mechanisms and models of somatic cell reprogramming. Nat Rev Genet. 2013;14(6):427-39.

8. Cacchiarelli D, Trapnell C, Ziller MJ, Soumillon M, Cesana M, Karnik R, Donaghey J, Smith ZD, Ratanasirintrawoot S, Zhang X, et al. Integrative analyses of human reprogramming reveal dynamic nature of induced pluripotency. Cell. 2015;162(2):412-24.

9. Hussein SM, Puri MC, Tonge PD, Benevento M, Corso AJ, Clancy JL, Mosbergen R, Li M, Lee DS, Cloonan N, et al. Genome-wide characterization of the routes to pluripotency. Nature. 2014:516(7530):198-206.

10. Subramanyam D, Blelloch R. From microRNAs to targets: pathway discovery in cell fate transitions. Curr Opin Genet Dev. 2011:21(4):498-503.

11. Huo JS, Zambidis ET. Pivots of pluripotency: the roles of non-coding RNA in regulating embryonic and induced pluripotent stem cells. Biochim Biophys Acta. 2013;1830(2):2385-94 
12. Guttman M, Donaghey J, Carey BW, Garber M, Grenier JK, Munson G, Young $G$, Lucas $A B, A c h R$, Bruhn $L$, et al. lincRNAs act in the circuitry controlling pluripotency and differentiation. Nature. 2011;477(7364):295-300.

13. Worringer KA, Rand TA, Hayashi Y, Sami S, Takahashi K, Tanabe K, Narita M, Srivastava D, Yamanaka S. The let-7/LIN-41 pathway regulates reprogramming to human induced pluripotent stem cells by controlling expression of prodifferentiation genes. Cell Stem Cell. 2014;14(1):40-52.

14. Kim DH, Marinov GK, Pepke S, Singer ZS, He P, Williams B, Schroth GP Elowitz MB, Wold BJ. Single-cell transcriptome analysis reveals dynamic changes in IncRNA expression during reprogramming. Cell Stem Cell. 2015;16(1):88-101.

15. Stadtfeld M, Apostolou E, Ferrari F, Choi J, Walsh RM, Chen T, Ooi SS, Kim SY, Bestor TH, Shioda T, et al. Ascorbic acid prevents loss of Dlk1-Dio3 imprinting and facilitates generation of all-iPS cell mice from terminally differentiated B cells. Nat Genet. 2012:44(4):398-405. S391-392

16. Stadtfeld M, Apostolou E, Akutsu H, Fukuda A, Follett P, Natesan S, Kono $T$, Shioda T, Hochedlinger K. Aberrant silencing of imprinted genes on chromosome 12qF1 in mouse induced pluripotent stem cells. Nature. 2010;465(7295):175-81

17. Liu L, Luo GZ, Yang W, Zhao X, Zheng Q, Lv Z, Li W, Wu HJ, Wang L, Wang $X J$, et al. Activation of the imprinted Dlk1-Dio3 region correlates with pluripotency levels of mouse stem cells. J Biol Chem. 2010;285(25): 19483-90.

18. Lin SP, Youngson N, Takada S, Seitz H, Reik W, Paulsen M, Cavaille J, Ferguson-Smith AC. Asymmetric regulation of imprinting on the maternal and paternal chromosomes at the Dlk1-Gtl2 imprinted cluster on mouse chromosome 12. Nat Genet. 2003;35(1):97-102.

19. Xu X, Smorag L, Nakamura T, Kimura T, Dressel R, Fitzner A, Tan X, Linke M, Zechner $U$, Engel $W$, et al. Dppa3 expression is critical for generation of fully reprogrammed iPS cells and maintenance of Dlk1-Dio3 imprinting. Nat Commun. 2015;6:6008

20. Das PP, Hendrix DA, Apostolou E, Buchner AH, Canver MC, Beyaz S, Ljuboja D, Kuintzle R, Kim W, Karnik R, et al. PRC2 is required to maintain expression of the maternal Gtl2-Rian-Mirg locus by preventing de novo DNA methylation in mouse embryonic stem cells. Cell Rep. 2015;12(9):1456-70.

21. Heinrich PC, Behrmann I, Muller-Newen G, Schaper F, Graeve L. Interleukin-6-type cytokine signalling through the gp130/Jak/STAT pathway. Biochem J. 1998;334(Pt 2):297-314.

22. Taga T, Kishimoto T. Gp130 and the interleukin-6 family of cytokines. Annu Rev Immunol. 1997;15:797-819.

23. Niwa H, Burdon T, Chambers I, Smith A. Self-renewal of pluripotent embryonic stem cells is mediated via activation of STAT3. Genes Dev. 1998; 12(13):2048-60.

24. Matsuda T, Nakamura T, Nakao K, Arai T, Katsuki M, Heike T, Yokota T. STAT3 activation is sufficient to maintain an undifferentiated state of mouse embryonic stem cells. EMBO J. 1999:18(15):4261-9.

25. Smith AG, Heath JK, Donaldson DD, Wong GG, Moreau J, Stahl M, Rogers D. Inhibition of pluripotential embryonic stem cell differentiation by purified polypeptides. Nature. 1988;336(6200):688-90.

26. Williams RL, Hilton DJ, Pease $S$, Willson TA, Stewart $C L$, Gearing DP, Wagner EF, Metcalf D, Nicola NA, Gough NM. Myeloid leukaemia inhibitory factor maintains the developmental potential of embryonic stem cells. Nature. 1988;336(6200):684-7.

27. Nichols J, Smith A. Naive and primed pluripotent states. Cell Stem Cell. 2009;4(6):487-92.

28. Yang J, van Oosten AL, Theunissen TW, Guo G, Silva JC, Smith A. Stat3 activation is limiting for reprogramming to ground state pluripotency. Cell Stem Cell. 2010;7(3):319-28.

29. Tang $Y$, Luo $Y$, Jiang Z, Ma Y, Lin CJ, Kim C, Carter MG, Amano T, Park J, Kish S, et al. Jak/Stat3 signaling promotes somatic cell reprogramming by epigenetic regulation. Stem Cells. 2012;30(12): 2645-56.

30. van Oosten AL, Costa $Y$, Smith A, Silva JC. JAK/STAT3 signalling is sufficient and dominant over antagonistic cues for the establishment of naive pluripotency. Nat Commun. 2012;3:817.

31. De Los Angeles A, Loh YH, Tesar PJ, Daley GQ. Accessing naive human pluripotency. Curr Opin Genet Dev. 2012;22(3):272-82.

32. Niwa H, Ogawa K, Shimosato D, Adachi K. A parallel circuit of LIF signalling pathways maintains pluripotency of mouse ES cells. Nature. 2009;460(7251):118-22.
33. Thompson JE, Cubbon RM, Cummings RT, Wicker LS, Frankshun R, Cunningham BR, Cameron PM, Meinke PT, Liverton N, Weng Y, et al. Photochemical preparation of a pyridone containing tetracycle: a Jak protein kinase inhibitor. Bioorg Med Chem Lett. 2002;12(8):1219-23.

34. Yang J, van Oosten AL, Theunissen TW, Guo G, Silva JC, Smith A. Stat3 activation is limiting for reprogramming to ground state pluripotency. Cell Stem Cell. 7(3):319-28.

35. Martello G, Bertone P, Smith A. Identification of the missing pluripotency mediator downstream of leukaemia inhibitory factor. EMBO J. 2013;32(19): 2561-74.

36. Ye S, Li P, Tong C, Ying QL. Embryonic stem cell self-renewal pathways converge on the transcription factor Tfcp2l1. EMBO J. 2013;32(19):2548-60.

37. Dennis G Jr, Sherman BT, Hosack DA, Yang J, Gao W, Lane HC, Lempicki RA DAVID: database for annotation, visualization, and integrated discovery. Genome Biol. 2003:4(5):P3.

38. Branzei D, Foiani M. Regulation of DNA repair throughout the cell cycle. Nat Rev Mol Cell Biol. 2008:9(4):297-308.

39. Spiess C, Meyer AS, Reissmann S, Frydman J. Mechanism of the eukaryotic chaperonin: protein folding in the chamber of secrets. Trends Cell Biol. 2004;14(11):598-604.

40. Kang CB, Hong Y, Dhe-Paganon S, Yoon HS. FKBP family proteins: immunophilins with versatile biological functions. Neurosignals. 2008;16(4): 318-25.

41. Gareau JR, Lima CD. The SUMO pathway: emerging mechanisms that shape specificity, conjugation and recognition. Nat Rev Mol Cell Biol. 2010;11(12): 861-71

42. Wang PJ, McCarrey JR, Yang F, Page DC. An abundance of X-linked genes expressed in spermatogonia. Nat Genet. 2001;27(4):422-6.

43. Soper SF, van der Heijden GW, Hardiman TC, Goodheart M, Martin SL, de Boer $\mathrm{P}$, Bortvin A. Mouse maelstrom, a component of nuage, is essential for spermatogenesis and transposon repression in meiosis. Dev Cell. 2008;15(2): 285-97.

44. Bolcun-Filas E, Hall E, Speed R, Taggart M, Grey C, de Massy B, Benavente R, Cooke HJ. Mutation of the mouse Syce1 gene disrupts synapsis and suggests a link between synaptonemal complex structural components and DNA repair. PLoS Genet. 2009;5(2):e1000393.

45. Bolcun-Filas E, Costa Y, Speed R, Taggart M, Benavente R, De Rooij DG, Cooke HJ. SYCE2 is required for synaptonemal complex assembly, double strand break repair, and homologous recombination. J Cell Biol. 2007;176(6): 741-7.

46. Marumoto T, Zhang D, Saya H. Aurora-a - a guardian of poles. Nat Rev Cancer. 2005;5(1):42-50.

47. Borlado LR, Mendez J. CDC6: from DNA replication to cell cycle checkpoints and oncogenesis. Carcinogenesis. 2008;29(2):237-43.

48. Liu Y, Tarsounas M, O'Regan P, West SC. Role of RAD51C and XRCC3 in genetic recombination and DNA repair. J Biol Chem. 2007;282(3):1973-9.

49. Esashi F, Christ N, Gannon J, Liu Y, Hunt T, Jasin M, West SC. CDKdependent phosphorylation of BRCA2 as a regulatory mechanism for recombinational repair. Nature. 2005:434(7033):598-604.

50. Thu YM, Bielinsky AK. MCM10: one tool for all-integrity, maintenance and damage control. Semin Cell Dev Biol. 2014;30:121-30.

51. Anderson EL, Baltus AE, Roepers-Gajadien HL, Hassold TJ, de Rooij DG, van Pelt AM, Page DC. Stra8 and its inducer, retinoic acid, regulate meiotic initiation in both spermatogenesis and oogenesis in mice. Proc Natl Acad Sci U S A. 2008:105(39):14976-80.

52. Suzuki H, Ahn HW, Chu T, Bowden W, Gassei K, Orwig K, Rajkovic A. SOHLH1 and SOHLH2 coordinate spermatogonial differentiation. Dev Biol. 2012;361(2):301-12.

53. Ko K, Tapia N, Wu G, Kim JB, Bravo MJ, Sasse P, Glaser T, Ruau D, Han DW Greber B, et al. Induction of pluripotency in adult unipotent germline stem cells. Cell Stem Cell. 2009;5(1):87-96.

54. Pek JW, Lim AK, Kai T. Drosophila maelstrom ensures proper germline stem cell lineage differentiation by repressing microRNA-7. Dev Cell. 2009;17(3): $417-24$

55. Chen $X, X u H$, Yuan P, Fang F, Huss M, Vega VB, Wong E, Orlov YL, Zhang $W$, Jiang J, et al. Integration of external signaling pathways with the core transcriptional network in embryonic stem cells. Cell. 2008;133(6):1106-17.

56. Wendt MK, Balanis N, Carlin CR, Schiemann WP. STAT3 and epithelialmesenchymal transitions in carcinomas. JAKSTAT. 2014;3(1):e28975.

57. Garg M. Epithelial-mesenchymal transition - activating transcription factors multifunctional regulators in cancer. World J Stem Cells. 2013;5(4):188-95. 
58. Groger CJ, Grubinger M, Waldhor T, Vierlinger K, Mikulits W. Meta-analysis of gene expression signatures defining the epithelial to mesenchymal transition during cancer progression. PLoS One. 2012;7(12):e51136.

59. Ventura A, Young AG, Winslow MM, Lintault L, Meissner A, Erkeland SJ, Newman J, Bronson RT, Crowley D, Stone JR, et al. Targeted deletion reveals essential and overlapping functions of the miR-17 through 92 family of miRNA clusters. Cell. 2008;132(5):875-86.

60. Greve TS, Judson RL, Blelloch R. microRNA control of mouse and human pluripotent stem cell behavior. Annu Rev Cell Dev Biol. 2013;29:213-39.

61. Lim LS, Loh YH, Zhang W, Li Y, Chen X, Wang Y, Bakre M, Ng HH, Stanton LW. Zic3 is required for maintenance of pluripotency in embryonic stem cells. Mol Biol Cell. 2007;18(4):1348-58.

62. Tesar PJ, Chenoweth JG, Brook FA, Davies TJ, Evans EP, Mack DL, Gardner RL, McKay RD. New cell lines from mouse epiblast share defining features with human embryonic stem cells. Nature. 2007;448(7150):196-9.

63. Brons IG, Smithers LE, Trotter MW, Rugg-Gunn P, Sun B, Chuva de Sousa Lopes SM, Howlett SK, Clarkson A, Ahrlund-Richter L, Pedersen RA, et al. Derivation of pluripotent epiblast stem cells from mammalian embryos. Nature. 2007;448(7150):191-5.

64. Ogawa K, Saito A, Matsui H, Suzuki H, Ohtsuka S, Shimosato D, Morishita Y, Watabe T, Niwa H, Miyazono K. Activin-Nodal signaling is involved in propagation of mouse embryonic stem cells. J Cell Sci. 2007;120(Pt 1):55-65.

65. Ying QL, Wray J, Nichols J, Batlle-Morera L, Doble B, Woodgett J, Cohen P, Smith $A$. The ground state of embryonic stem cell self-renewal. Nature. 2008:453(7194):519-23.

66. Allis CD, Berger SL, Cote J, Dent $\mathrm{S}$, Jenuwien T, Kouzarides T, Pillus L, Reinberg D, Shi Y, Shiekhattar R, et al. New nomenclature for chromatinmodifying enzymes. Cell. 2007;131(4):633-6.

67. Anzick SL, Kononen J, Walker RL, Azorsa DO, Tanner MM, Guan XY, Sauter G, Kallioniemi OP, Trent JM, Meltzer PS. AIB1, a steroid receptor coactivator amplified in breast and ovarian cancer. Science. 1997;277(5328):965-8.

68. Takeshita A, Cardona GR, Koibuchi N, Suen CS, Chin WW. TRAM-1, a novel 160-kDa thyroid hormone receptor activator molecule, exhibits distinct properties from steroid receptor coactivator-1. J Biol Chem. 1997;272(44): 27629-34.

69. Fragola G, Germain PL, Laise P, Cuomo A, Blasimme A, Gross F, Signaroldi E, Bucci G, Sommer C, Pruneri G, et al. Cell reprogramming requires silencing of a core subset of polycomb targets. PLoS Genet. 2013;9(2):e1003292.

70. Gao Y, Chen J, Li K, Wu T, Huang B, Liu W, Kou X, Zhang Y, Huang H, Jiang $Y$, et al. Replacement of Oct4 by Tet1 during iPSC induction reveals an important role of DNA methylation and hydroxymethylation in reprogramming. Cell Stem Cell. 2013;12(4):453-69.

71. Olariu V, Lovkvist C, Sneppen K. Nanog, Oct4 and Tet1 interplay in establishing pluripotency. Sci Rep. 2016;6:25438.

72. Weinberger L, Ayyash M, Novershtern N, Hanna JH. Dynamic stem cell states: naive to primed pluripotency in rodents and humans. Nat Rev Mol Cell Biol. 2016;17(3):155-69.

73. Hustedt N, Durocher D. The control of DNA repair by the cell cycle. Nat Cell Biol. 2016, 19:1):1-9.

74. Kiger AA, Jones DL, Schulz C, Rogers MB, Fuller MT. Stem cell self-renewal specified by JAK-STAT activation in response to a support cell cue. Science. 2001;294(5551):2542-5.

75. Wawersik M, Milutinovich A, Casper AL, Matunis E, Williams B, Van Doren M. Somatic control of germline sexual development is mediated by the JAK STAT pathway. Nature. 2005;436(7050):563-7.

76. Lopez-Onieva L, Fernandez-Minan A, Gonzalez-Reyes A. Jak/Stat signalling in niche support cells regulates dpp transcription to control germline stem cell maintenance in the drosophila ovary. Development. 2008;135(3):533-40.

77. Issigonis M, Tulina N, de Cuevas M, Brawley C, Sandler L, Matunis E. JAKSTAT signal inhibition regulates competition in the drosophila testis stem cell niche. Science. 2009;326(5949):153-6.

78. Sobinoff AP, Sutherland JM, McLaughlin EA. Intracellular signalling during female gametogenesis. Mol Hum Reprod. 2013;19(5):265-78.

79. Spradling A, Fuller MT, Braun RE, Yoshida S. Germline stem cells. Cold Spring Harb Perspect Biol. 2011;3(11):a002642.

80. Yamaji M, Seki Y, Kurimoto K, Yabuta Y, Yuasa M, Shigeta M, Yamanaka K, Ohinata Y, Saitou M. Critical function of Prdm14 for the establishment of the germ cell lineage in mice. Nat Genet. 2008:40(8):1016-22.

81. Raymond CS, Murphy MW, O'Sullivan MG, Bardwell VJ, Zarkower D. Dmrt1, a gene related to worm and fly sexual regulators, is required for mammalian testis differentiation. Genes Dev. 2000;14(20):2587-95.
82. Wu J, Platero-Luengo A, Sakurai M, Sugawara A, Gil MA, Yamauchi T, Suzuki K, Bogliotti YS, Cuello C, Morales Valencia M, et al. Interspecies Chimerism with mammalian pluripotent stem cells. Cell. 2017;168(3):473-86. e415

83. Najm FJ, Chenoweth JG, Anderson PD, Nadeau JH, Redline RW, McKay RD, Tesar PJ. Isolation of epiblast stem cells from preimplantation mouse embryos. Cell Stem Cell. 2011;8(3):318-25.

84. Yang Y, Liu B, Xu J, Wang J, Wu J, Shi C, Xu Y, Dong J, Wang C, Lai W, et al. Derivation of pluripotent stem cells with in vivo embryonic and extraembryonic potency. Cell. 2017;169(2):243-57. e225

85. Waghray A, Saiz N, Jayaprakash AD, Freire AG, Papatsenko D, Pereira CF, Lee DF, Brosh R, Chang B, Darr H, et al. Tbx3 controls Dppa3 levels and exit from pluripotency toward mesoderm. Stem Cell Rep. 2015;5(1):97-110.

86. Jiang Z, Sun J, Dong H, Luo O, Zheng X, Obergfell C, Tang Y, Bi J, O'Neill R, Ruan $Y$, et al. Transcriptional profiles of bovine in vivo pre-implantation development. BMC Genomics. 2014;15:756.

87. Trapnell C, Pachter L, Salzberg SL. TopHat: discovering splice junctions with RNA-Seq. Bioinformatics. 2009;25(9):1105-11.

88. Trapnell C, Roberts A, Goff L, Pertea G, Kim D, Kelley DR, Pimentel H, Salzberg SL, Rinn JL, Pachter L. Differential gene and transcript expression analysis of RNA-seq experiments with TopHat and cufflinks. Nat Protoc. 2012;7(3):562-78.

89. Huang d W, Sherman BT, Lempicki RA. Systematic and integrative analysis of large gene lists using DAVID bioinformatics resources. Nat Protoc. 2009; 4(1):44-57.

\section{Submit your next manuscript to BioMed Central and we will help you at every step:}

- We accept pre-submission inquiries

- Our selector tool helps you to find the most relevant journal

- We provide round the clock customer support

- Convenient online submission

- Thorough peer review

- Inclusion in PubMed and all major indexing services

- Maximum visibility for your research

Submit your manuscript at www.biomedcentral.com/submit

) Biomed Central 